\title{
Psoriasis pathophysiology and impact on life
}

\section{Tariq Alharbi ${ }^{1 *}$, Ali Alzahrani ${ }^{2}$, Afaf Hakami ${ }^{3}$, Adeeb Almohammadi ${ }^{4}$, Abdullah Alsaadi ${ }^{4}$, Norah Bin Magbel $^{1}$, Najd AlJuaid ${ }^{4}$, Haneen Almasoudi ${ }^{5}$, Taif Alruwaili ${ }^{6}$, Sarah Alkhezzi ${ }^{7}$}

\author{
${ }^{1}$ Almaarefa Colleges for Science and Technology, Riyadh, KSA \\ ${ }^{2}$ King Abdulaziz University, Rabigh, KSA \\ ${ }^{3}$ Jazan University, Jizan, KSA \\ ${ }^{4}$ Ibn Sina National College for Medical Studies, Jeddah, KSA \\ ${ }^{5}$ Umm AlQura University, Mecca, KSA \\ ${ }^{6}$ Hail University, Hail, KSA \\ ${ }^{7}$ Qassim University, Qassim, KSA
}

Received: 09 July 2018

Accepted: 25 July 2018

\section{*Correspondence:}

Dr. Tariq Alharbi,

E-mail: tariq7-7-7@hotmail.com

Copyright: (C) the author(s), publisher and licensee Medip Academy. This is an open-access article distributed under the terms of the Creative Commons Attribution Non-Commercial License, which permits unrestricted non-commercial use, distribution, and reproduction in any medium, provided the original work is properly cited.

\begin{abstract}
Psoriasis is a chronic skin disorder with various morphology, distribution, severity, and course of disease. The goal of the management is to control disease activity to a level that allows a sufficient quality of life with minimal toxicity from the treatment itself. Unfortunately, $25 \%$ of patients suffering from psoriasis experience major psychological distress. Its chronic nature with associated comorbidities has a negative impact on quality of life. We conducted this review using a comprehensive search of MEDLINE, PubMed and EMBASE from January 1996 to March 2017. The following search terms were used: psoriasis, pathogenesis of psoriasis, psoriasis management, quality of life in psoriasis patients. We tried to study the pathogenesis, and management of psoriasis, and understand its effect on the quality of life of patients. Due to the chronic nature and its obvious visibility on skin, many patients suffer from major psychological adverse effects. In majority of the time the health care providers fail to recognize or treat such comorbidities. Healthcare providers must be educated to provide patients with ways to tackle issues with multiple aspects, including psychological, in the management plan for better results.
\end{abstract}

Keywords: Psoriasis, Chronic skin disorders, Pathogenesis of psoriasis, Management of psoriasis, Quality of life in psoriatic patients

\section{INTRODUCTION}

Psoriasis is an important dermatological disease that is characterized by squamous papules that differ in severity, morphology, distribution, and progression among patients. When dealing a case of possible psoriasis, the differential diagnosis should include lichen planus, tinia infections, and pityriasis rosea. Psoriasis lesions can be distinguished by their red, round shape which makes well-circumscribed plaques or papules. These plaques and papules are characterized by having a characteristic silver white scale. Lesions of psoriasis are usually symmetrically distributed on the elbows, knees, scalp, lumbosacral area, and body folds. They can also sometimes occur in areas of trauma or injury, which is called 'Koebner's phenomenon'. Untreated psoriasis cases, or cases with a progressive course may present as generalized exfoliative erythroderma that sometimes, especially in psoriatic arthritis, can involve the nails. Psoriatic lesions on the tongue or elsewhere on the oral mucosa could sometimes be also found in cases of sporadic psoriasis. ${ }^{1}$ 
Usually, overall survival and mortality are not affected by a diagnosis of psoriasis. However, it can negatively affect the quality of life of the patient, causing several negative net effects. Its chronic and incurable nature make it carry significant morbidity to patients. About a quarter of patients with psoriasis report psychological distress and at least one major psychological event. They also largely have the feeling of social stigmatism. However, these significant negative effects are many times underestimated by treating physicians, making the case worse sometimes. ${ }^{2}$ In this study, we aim to discuss psoriasis and its etiologies, types, management, and effects on patients' quality of life.

\section{METHODS}

\section{Data sources and search terms}

We conducted this review using a comprehensive search of MEDLINE, PubMed and EMBASE, from January 1996 to March 2017. The following search terms were used: psoriasis, pathogenesis of psoriasis, psoriasis management, quality of life in psoriasis patients

\section{Data extraction}

Two reviewers have independently reviewed the studies, abstracted data and disagreements were resolved by consensus. Studies were evaluated for quality and a review protocol was followed throughout.

This study was done after approval of Review Board of King Abdulaziz University.

\section{TYPES}

Psoriasis can vary greatly in morphology, in its distribution as well as severity. In terms of morphology they range from small tear shaped papules known as guttate psoriasis, to pustules which are known as pustular psoriasis, erythrodermic psoriasis which is generalized scaly erythema. ${ }^{3}$ Psoriasis can also be classified as localized or widespread, and based upon the course such as chronic, stable or acute rapidly progressing type. In this review, we are discussing the various types based upon their clinical presentation, as listed below:

\section{Plaque psoriasis}

This is the most common form of psoriasis in which patients present with sharply bordered, oval to round shaped plaques of the size of a coin. A white blanching ring, which is known as a Woronoff's ring, can be noticed in the skin around the plaque. The plaque can later extend peripherally and acquire several configurations as: ${ }^{4}$

- Psoriasis gyrata — curved linear patterns

- Annular psoriasis-ring-like lesions with central clearing
- Psoriasis follicularis-small scaly papules at the pilo-sebaceous follicles opening

\section{Flexural (inverse) psoriasis}

This type of psoriasis affects the flexures, especially infra-mammary, perineal, and axillary. It is different morphologically from the other plaques that occur on the trunk and limbs. Flexural lesions are not scaly and are well-marked with red and shiny appearance. Sometimes they can be confused with candida and other dermatophyte infections. ${ }^{5}$

\section{Erythroderma}

Skin involvement by active psoriasis is called erythroderma and can take either of two forms. First, chronic plaque psoriasis could progressively increase and develop as confluent and extensive. Second, erythroderma can indicate an underlying unstable psoriasis which was triggered by infection, certain drugs, tar, or sudden corticosteroid withdrawal. Erythroderma can harm the capability of the skin to thermo regulate, leading to hypothermia, high output heart failure, and metabolic disturbances such as anemia due to loss of iron, folic acid, and vitamin B12, and folic acid, as well as hypo-albuminemia. ${ }^{6}$

\section{Generalized pustular psoriasis}

Generalized pustular psoriasis is not very common but indicates an active and unstable disease. It can be triggered by withdrawal of strong topical or systemic corticosteroids as well as infections. The patient presents with fever and red, inflamed, and painful skin dotted with sterile pustules, which may coalesce to form sheets. Such patients often need to hospital admission for appropriate management. ${ }^{7}$

\section{Palmoplantar pustulosis}

Palmoplantar pustulosis can begin as sterile, yellow pustules surrounded by erythema and scaling. They often affect the palms and soles. The pustules are painful and transform in to dark brown coloration with scaly crust stuck to it. Palmoplantar pustulosis is usually associated with psoriatic nail involvement. Around $25 \%$ of cases are associated with classic psoriasis vulgaris. This assumption is based upon the genetic studies displaying no link with HLA-Cw6 or other markers on chromosome $6 \mathrm{p}$ - which are linked to guttate psoriasis and chronic plaque. ${ }^{8}$ The demographics of palmoplantar pustulosis are markedly different from those of chronic plaque psoriasis in that it more recurrently affects women with a ratio of 8.2 to 1 , presents usually between the ages of 20-60 years, and has a strong correlation with smoking history, either recent or in past. ${ }^{9}$ 


\section{Psoriatic nail disease}

Compared to toenails, fingernails are more frequently affected. The most common presentation is minor pits in the nail plate, as a result of flawed nail formation in the proximal part of the nail bed. The nail could also separate from the bed at its distal or lateral attachments, as in onycholysis. Orange-yellow can be noticed underneath the nail plate and are named as oil spots. Additionally, the nail plate can turn thick, discolored and dystrophic. Yellow, keratinous substance can be found under the nail plate and is known as subungual hyperkeratosis. ${ }^{10}$

\section{PATHOGENESIS}

The occurrence of psoriasis can be explained by many reasons. These include drugs, trauma, infections, and imiquimod (a TLR7 agonist), which is a topical biological reaction modifier. Several previous reports have suggested that the topical application of imiquimod may trigger the development of cause psoriasiform skin inflammation. This is thought to be activated by IL-23 and IL-17, causing significant damage in the skin that usually ends in necrosis and the secretion of AMP LL37 by keratinocytes. Complexes that include DNA/LL37 will later bind to TLR9 which is present intra-cellularly within the plasmacytoid dendritic cells. Consequently, type I interferons IFN- $\alpha$ and $-\beta$ are stimulated, produced and secreted. This will cause the activation of plasmacytoid DCs by LL37/RNA complexes and TLR7. On the other hand TLR8 will help in the activation of myeloid DCs. ${ }^{11}$ After these complex steps, myeloid DCs are activated, which will lead to the activation of $\mathrm{T}$ cells and the secretion of cytokines that will cause psoriasis. Some researchers suggested the presence of extracellular DNA in the epidermis along with neutrophils, which largely supports this theory of psoriasis development. ${ }^{12}$

\section{Genetic factors}

When the genome of humans was studied, nine loci were found to be associated with the development of psoriasis (PSORS1-9). PSORS-1 is present on chromosome 6p2, and is a part of the major histocompatibility complex. It is considered the most important determiner of psoriasis, and is, alone, responsible for up to $50 \%$ of the risk. Some studies have found that some loci associated with psoriasis are mutually associated with other inflammatory and autoimmune diseases including multiple sclerosis, atopic dermatitis, inflammatory bowel disease, and type 1 diabetes mellitus. We can conclude from these findings that similar mechanisms may be involved in psoriasis as well as all these mentioned diseases. ${ }^{13}$

Other genes can also show mutations that are associated with the development of psoriasis. An example of this is the IL-1F5 gene that codes for the IL-36Ra protein. This protein is an anti-inflammatory protein that works as an antagonist for the IL-1F9 protein. Therefore, a mutation on this gene will potentially cause alterations in the formation and secretion of IL-36Ra protein and thus an increased activity of IL-1F9. This will cause a stronger activation of NF- $\mathrm{NB}$ and MAPK. Studies that compared patients with psoriasis with healthy individuals revealed increased levels of NF- $\mathrm{BB}$ cytokines in mononuclear cells of patients. This led to the conclusion that when IL36RN losses its function, this will strongly predict the development of generalized pustular psoriasis. ${ }^{14}$

\section{Immunological and environmental factors}

Both innate and acquired immune immunity mechanisms have been suggested to be involved in the pathogenicity of psoriasis. In these cases, tumor necrosis factor $\alpha$ and interferon $\alpha$, along with other cytokines are released when an environmental trigger, like infection, trauma, stress, and drugs, is present. The fact that infliximab (a monoclonal antibody against TNF $\alpha$ ) provides treatment to psoriasis, strongly supports this hypothesis, and emphasizes on the effects of both innate and acquired immunity in the disease ${ }^{15}$.

\section{TREATMENT}

Treatment of psoriasis usually aims at the reduction of severity of symptoms to the level that allows normal (as much as possible) quality of life. On the other hand, toxicity associated with the treatment should also be considered and minimized. Common methods for relieving symptoms include tonsillectomy and the use of antibiotics. However, solid evidence supporting these interventions is lacking. Other therapies include phototherapy, topical therapy, systemic therapy with immunosuppressant drugs, and the use of biological agents. $^{16}$

\section{Topical therapy}

Usually, topical therapy is associated with poor compliance in most patients. Agents approved for topical therapy in psoriasis include tar, tazarotene, corticosteroids, vitamin D, and dithranol. Previous studies have concluded that vitamin D analogues are similar in efficacy to highly potent corticosteroids, and are superior to dithranol. Moreover, they were found to be associated with less risk of side effects. Of available vitamin $\mathrm{D}$ analogues, calcipotriol has been found to be the most effective. Moreover, its use with a potent corticosteroid is even more effective. ${ }^{17}$ On real-life settings, the use of topical corticosteroids is largely common due to their efficacy and rapid onset of action. Corticosteroids with mild to moderate potency are usually used in cases of psoriasis involving the face or body flexures. Its use with calcipotriol will further increase the response rate and decrease remission rates. it can moreover, decrease the risk of developing side effects that include skin atrophy, and rebound psoriasis. ${ }^{18}$ 


\section{Phototherapy and systemic treatments}

The short-term efficacy of ultraviolet B-light phototherapy and PUVA photochemotherapy for the treatment and relief of psoriasis is well-documented and proven with several clinical trials. However, its safety of these methods is not well-studied, and existing literature does not provide a solid evidence regarding its safety and relative efficacy when compared to other interventions. Another important aspect where there is lack of evidence is the outcomes when this kind of intervention is stopped or given intermittently, and the length of remission periods in these cases before relapse occurs. ${ }^{19}$ To reduce the risks of drugs toxicity, long-term management tends to avoid the use of systemic treatment, provide drug holidays, and give a combination of drugs to allow for dose reduction. When dealing with patients who suffer from severe, refractory psoriasis, a multidisciplinary team of specialists should be involved to provide the best treatment protocol. ${ }^{20}$

Up till now, the best drug to treat moderate or severe psoriasis is thought to be methotrexate. It is also effective in cases with nails or joints involvement, and provides efficacy and symptoms relief for relatively long periods of time. Studies on methotrexate show that about three quarters of patients with psoriasis will show an improvement in symptoms that can reach up to $50 \%$. However, limitations for methotrexate use include its long term adverse events, most severely, silent liver cirrhosis, which although rare, requires routine liver biopsies. Recently, detection of this serious adverse event have been done by the routine screening of serum procollagen III every three months. ${ }^{21}$

\section{Biological treatments}

Biological therapy is the use of drugs that interact with the basic mechanisms of the disease pathophysiology. Most important biological drugs in the treatment of psoriasis are agents that inhibit TNF $\alpha$ (infliximab, adalimumab, etanercept), and agents that inhibit antigen presenting cells or $\mathrm{T}$ cells (efalizumab). ${ }^{22}$ Despite being given intravenously, the use of these drugs have largely increased lately. The reason behind this is likely to be the relative failure of other therapies in achieving patients' satisfaction. These drugs are mainly associated with a high risk of developing infections, especially tuberculosis with the use of anti TNF drugs. Moreover, the risk of treatment failure can still occur. ${ }^{23}$

\section{IMPACT ON QUALITY OF LIFE}

\section{Psychosocial effect}

When discussing impaired quality of life in psoriasis patients, it is essential to address social, functional, and psychological aspects. Symptoms of psoriasis are essentially related to the skin and may include scaling, itching, bleeding, and nail involvement. Therapies also cause dis-comfort to patients as it can cause strange odor, long duration of use, mess, and inconvenience. Moreover, patients will need long-term observation and follow-up, and will most likely develop skin diseases and disfigurements, which will later affect their relationships and social encounters, and cause them to have relatively low self-esteem. ${ }^{24}$

About a quarter of patients who have psoriasis have suffered from psychological stress at least once in their life. They also felt social stigmatism and exclusion. Usually, clinicians underestimate this problem and fail to understand the impact and burden of the disease on patients. Moreover, it has been found that even when clinicians correctly recognize the psychological distress, more than two thirds of cases do not receive proper therapy. Proper psychological intervention is essential and will largely effect management outcomes. a previous study has examined the role of cognitive behavioral therapy when given along with standard treatment to psoriasis patients. They concluded that it significantly led to decrease in levels of stress, anxiety, disability, depression, and extent of the disease. ${ }^{24}$

\section{Associated comorbidity: cardiovascular disease and cancer}

Patients who have severe psoriasis have been found to have about two or three folds higher risk of cardiovascular mortality. This increased risk is thought to be due to several potential risk factors. Psoriasis patients are also at double the risk of being obese when compared to other populations. Researchers think that this higher obesity rates is due to sedentary life styles that patients develop after their psoriasis become more severe. Smoking and alcohol have also been found to increase the risk of psoriasis. As we previously mentioned, patients with psoriasis may have higher levels of stress, this may be a predisposing factor and a trigger for relapses. In addition, severe psoriasis have been linked with higher incidence of hyperlipidemia. This may be a long-term side effect of psoriasis treatment. Chronic inflammation has also been linked to psoriasis along with other diseases like rheumatoid arthritis and lupus. ${ }^{25}$

\section{CONCLUSION}

Psoriasis is an important dermatological disorder that occurs due to complex mechanisms and pathogenesis. A variety of treatment options is present, which include topical and systemic therapies. However, the chronic progressive nature of the disease still causes significant stress and psychological effects to patients. These aspects are usually under-estimated and not properly recognized by clinicians which will cause further disability to patients. Clinicians should be further educated and trained on how to deal with psoriasis patients.

\section{Funding: No funding sources \\ Conflict of interest: None declared \\ Ethical approval: Not required}




\section{REFERENCES}

1. Di Meglio P, Villanova F, Nestle F. O. Psoriasis. Cold Spring Harb Perspect Med. 2014: 4.

2. Bhosle MJ, Kulkarni A, Feldman SR, Balkrishnan R. Quality of life in patients with psoriasis. Health Qual Life Outcomes. 2006;4:35.

3. Sarac G, Koca TT, Baglan T. A brief summary of clinical types of psoriasis. North Clin Istanb. 2016;3:79-82.

4. Langley RG, Krueger GG, Griffiths CE. Psoriasis: epidemiology, clinical features, and quality of life. Ann Rheum Dis. 2005;64(2):18-25.

5. Guglielmetti A, Conlledo R, Bedoya J, Ianiszewski $\mathrm{F}$, Correa J. Inverse psoriasis involving genital skin folds:successful therapy with dapsone. Dermatol Ther (Heidelb). 2012;2:15.

6. Singh RK, Lee KM, Ucmak D, Brodsky M, Atanelov Z, Farahnik B, et al. Erythrodermic psoriasis:pathophysiology and current treatment perspectives. Psoriasis (Auckl). 2016;6:93-104.

7. Benjegerdes KE, Hyde K, Kivelevitch D, Mansouri B. Pustular psoriasis: pathophysiology and current treatment perspectives. Psoriasis (Auckl). 2016;6:131-44.

8. Elahmed HH. Rapid improvement of palmoplantar psoriasis after cessation of smoking. Sultan Qaboos Univ Med J. 2013;13:188-9.

9. Miceli A, Schmieder GJ. Palmoplantar Psoriasis. 2018.

10. Schons KR, Knob CF, Murussi N, Beber AA, Neumaier W, Monticielo OA. Nail psoriasis:a review of the literature. An Bras Dermatol. 2014;89:312-7.

11. Krueger G, Ellis CN. Psoriasis--recent advances in understanding its pathogenesis and treatment. J Am Acad Dermatol. 2005;53:S94-100.

12. Afshar M, Martinez AD, Gallo RL, Hata TR. Induction and exacerbation of psoriasis with Interferon-alpha therapy for hepatitis $\mathrm{C}$ : a review and analysis of 36 cases. J Eur Acad Dermatol Venereol. 2013;27:771-8.

13. Prieto-Perez R, Cabaleiro T, Dauden E, Ochoa D, Roman M, Abad-Santos F. Genetics of psoriasis and pharmacogenetics of biological drugs. Autoimmune Dis. 2013;2013:613086.

14. Clarke P. Psoriasis. Aust Fam Physician. 2011;40:468-73.
15. Martin DA, Towne JE, Kricorian G, Klekotka P, Gudjonsson JE, Krueger JG, et al. The emerging role of $\mathrm{IL}-17$ in the pathogenesis of psoriasis: preclinical and clinical findings. J Invest Dermatol. 2013;133:17-26.

16. Dattola A, Del Duca E, Saraceno R, Gramiccia T, Bianchi L. Safety evaluation of apremilast for the treatment of psoriasis. Expert Opin Drug Saf. 2017;16:381-5.

17. Samarasekera EJ, Sawyer L, Wonderling D, Tucker $\mathrm{R}$, Smith CH. Topical therapies for the treatment of plaque psoriasis: systematic review and network meta-analyses. Br J Dermatol. 2013;168:954-67.

18. Katz U, Shoenfeld Y, Zakin V, Sherer Y, Sukenik S. Scientific evidence of the therapeutic effects of dead sea treatments:a systematic review. Semin Arthritis Rheum. 2012;42:186-200.

19. Asztalos ML, Heller MM, Lee ES, Koo J. The impact of emollients on phototherapy: a review. J Am Acad Dermatol. 2013;68:817-24.

20. Halverstam CP, Lebwohl M. Nonstandard and offlabel therapies for psoriasis. Clin Dermatol. 2008;26:546-53.

21. Boffa MJ, Chalmers RJ. Methotrexate for psoriasis. Clin Exp Dermatol. 1996;21:399-408.

22. Sivamani RK, Correa G, Ono Y, Bowen MP, Raychaudhuri SP, Maverakis E. Biological therapy of psoriasis. Indian J Dermatol. 2010;55:161-70.

23. Branisteanu DE, Voicu CM, Cretu A, Dimitriu A, Luca MC, Salavastru CM. Adverse reactions of biological therapy for psoriasis. Rev Med Chir Soc Med Nat Iasi. 2015;119:38-44.

24. Parrish L. Psoriasis: symptoms, treatments and its impact on quality of life. Br J Community Nurs. 2012;17:524-8.

25. Parisi R, Symmons DP, Griffiths CE, Ashcroft DM, Identification, Management of Psoriasis et al. Global epidemiology of psoriasis:a systematic review of incidence and prevalence. J Invest Dermatol. 2013;133:377-85.

Cite this article as: Alharbi T, Alzahrani A, Hakami A, Almohammadi A, Alsaadi A, Magbel NB, et al. Psoriasis pathophysiology and impact on life. Int $\mathrm{J}$ Community Med Public Health 2018;5:3663-7. 